\title{
INTRODUCTION TO THE NEW VOLUME
}

Having introduced some changes in our editorial policy with regard to the contents and the distribution of our journal, we think it justified to explain to our readers the reasons which have induced us to make these alterations.

During the years of its existence, under the influence of the vigorous development of many disciplines, the programme of the journal has been altered from time to time. The Unity of Science Forum, which was included in Synthese before the Second World War, aimed at stressing the logical structure of the special sciences considered in relation to one another. It dealt especially with the logic of science, the history of science, the sociology of science and 'the' general theory of signs. In recent years these fields have been greatly enlarged and intensively cultivated.

New methodological problems have arisen, old ones have been rejected or are re-formulated. It seems to us that the behavioural sciences are still gaining ground, although within the science of man the gap between the physical and the social approach is more and more sharply outlined. And the question of the meta-observer, the analysis of the role of the observer in a theory, has urged itself upon the research-worker.

Evidently the social sciences are still in search of a unified methodology and a unified terminology. To our mind, the science of man, in aiming at its own methodological exactness, should beware of the pseudoexactness resulting from a blind imitation of mathematical and physical procedures. In pragmatistically oriented groups of scientists in the U.S.A., in France, in Great-Britain, in some Norwegian groups of scientists and in the circles of the Dutch significists the opinion has been held for many years that the linguistic, and also the non-verbal form of evaluations is accessible to a scientific handling, and some recent works published in this field of science will have convinced many research workers of the rightness of the conception that such an approach is not only possible, but that investigations of this kind can be carried out successfully. Moreover, the idea of a multidisciplinary approach in this field has found more and more acceptance. Besides statistics, other procedures like factor analysis and scaling techniques have been introduced as a means of measuring values.

In this connection we cannot pass by in silence the endeavours which 
have been made recently to build up a genetic epistemology on an empirical basis, studied likewise by groups of scientists of interdisciplinary fields, and the progress which has been made in the field of linguistics by those specialists who have successfully applied the mathematical theory of communication to linguistics.

In the light of all these new scientific activities, we have thought it useful to adapt our journal to the new situation, as outlined above and, thanks to the support from an official institution in The Netherlands, we have been enabled to renew also the management of our journal, which henceforth will be published quarterly, at regular intervals.

THE EDITORS 